\title{
Pancreatic necrosis: a challenging complication of acute pancreatitis
}

\begin{abstract}
Pancreatic necrosis is a complex and challenging complication of severe acute pancreatitis and it occurs in 15 to $25 \%$ of patients with acute pancreatitis. It represents one of the most serious complications of severe acute pancreatitis with a mortality rate of 20 to $30 \%$. Majority of necrosis are sterile and can be managed conservatively. However, approximately $30 \%$ of pancreatic necrosis develop infection and need an invasive intervention due to aggressive course. A minimal invasive step-up approach using percutaneous catheter drainage or endoscopic transluminal drainage is currently replacing more invasive open necrosectomy. This review provides an overview of patho-physiology, clinical feature and management of pancreatic necrosis.
\end{abstract}

Volume 6 Issue I - 2018

\author{
Mahendra Singh,' Sachin Kathuria,' Ashish \\ Saxena, ${ }^{2}$ Lovekesh kumar, ${ }^{3}$ Saurabh Jain,' \\ Shahnawaz Rasool' \\ 'Depatment of Urology, Sir Ganga Ram Hospital, India \\ ${ }^{2}$ Deptartment of Surgery, Hindu Rao Hospital, India \\ ${ }^{3}$ Deptartment of Surgery, MAMC, India
}

Correspondence: Mahendra Singh, Department of Urology, Sir Ganga Ram Hospital, Delhi, India, Pin I I0060,

Email dr.mahil II8@gmail.com

Received: January 8, 2018 | Published: January 18, 2018

\section{Introduction}

Pancreatic necrosis (PN) is the presence of focal or diffuse nonviable pancreatic parenchyma or peripancreatic fat. ${ }^{1,2}$ Pancreatic necrosis is a complication of severe acute pancreatitis (SAP) and it occurs in 15 to $25 \%$ of patients with acute pancreatitis. ${ }^{1}$ It represents one of the most serious complications of SAP with a mortality rate of 20 to $30 \% .{ }^{1,2}$ Despite having an enormous impact upon the well being of individual and health service providers alike, the management of necrotizing pancreatitis is a highly controversial topic and continues to be an issue of debate. Considering the large number of cases of pancreatitis, one encounters in day to day practice, it is essential to understand the pathology, clinical features, investigations and the recent trends in management of pancreatic necrosis.

\section{Pathophysiology}

As per current understanding, acute pancreatitis is triggered by obstruction of pancreatic duct and pancreatic acinar cells constitute the seat of injury. ${ }^{2}$ Acute necrotizing pancreatitis results from premature activation of pancreatic enzymes within the acinar cells of pancreas. ${ }^{2,3}$ Colocalization hypothesis states that premature activation of enzymes occurs because of the action of lysosomes upon zymogen granules inside the acinar cells. ${ }^{4}$ Cathepsin B secreted by lysosomes play an important role in the process. This fact has been established by in vivo studies upon the Cathepsin B knockout mice. ${ }^{4}$ Prematurely activated pancreatic enzymes induce the auto-digestion of pancreatic parenchyma leading to pancreatic and peri-pancreatic fat necrosis and an associated inflammatory reaction. ${ }^{2,3}$ The extent of these changes is directly related to the severity of an attack. ${ }^{3}$ In mild cases, interstitial edema with infiltration of inflammatory cells is seen, while severe cases are characterized by extensive necrosis, thrombosis of intra-pancreatic vessels, vascular disruption and intra-parenchymal hemorrhage. ${ }^{2,3}$ About two third of pancreatic necrosis cases occur in the first week of SAP and are associated with systemic inflammatory response and early multi-organ dysfunction, and extension of necrosis to more than $50 \%$ of the pancreas. ${ }^{3}$ The patients who survive this initial phase of systemic insult are at risk for secondary infection of peri-pancreatic fluid collections after a few weeks of onset.

\section{Clinical presentation}

Symptoms of pancreatic necrosis mimic those of acute or chronic pancreatitis, of which the most common is sudden excruciating pain in the upper abdomen. ${ }^{2,5}$ It may also involve the lower chest or the lower abdomen. Such pain is stab like in nature and radiates to the back. It is often worse when lying down but may feel less intense when sitting up or bending over., ${ }^{2,5}$ Other symptoms include relentless nausea and vomiting which persist even after emptying of stomach. ${ }^{2,3}$ Fever, tachycardia and tachypnoea are prominent owing to the release of inflammatory mediators. ${ }^{5}$ Third space loss of fluids results in hypovolemia and hypotension. ${ }^{5}$ In most severe cases retroperitoneal hemorrhage is a usual occurrence, which manifests itself as Grey Turner's sign, Cullen's sign, and Fox sign, respectively denoting the ecchymosis in flanks, peri-umbilical region and inguinal region. ${ }^{5,6}$ The revised Atlanta classification divides acute pancreatitis into two distinct phases: An early phase that occurs within the $1^{\text {st }}$ week of onset of disease; and a late phase that takes place after the $1^{\text {st }}$ week of onset. ${ }^{7}$ During the $1^{\text {st }}$ week, the pathologic conditions progress from early inflammation of pancreas with variable degrees of peripancreatic edema and ischemia to resolution or to permanent necrosis and liquefaction. ${ }^{7,8}$ In early phase, severity is entirely based on clinical parameters, because the need for treatment in this phase is determined primarily by the presence or absence of organ failure caused by systemic inflammatory response syndrome and much less by morphologic findings involving the pancreas and peripancreatic areas. ${ }^{7}$ Patients with organ failure that last more than 48hours or death are considered to have SAP. Mild acute pancreatitis in the first phase is defined as organ failure that resolves in 48 hours. ${ }^{7}$ The late 
phase begins after the $1^{\text {st }}$ week, may extend for weeks to months, and is characterized by increasing necrosis, infection, and persistent multiorgan failure. ${ }^{7,8}$

Clinical progression of acute pancreatitis can help in making diagnosis of acute pancreatitis. ${ }^{9}$ Clinical progression of disease can be abortive, slowly or quickly progressive. ${ }^{9}$ At abortive passing the process is associated with acute edema of pancreas with convalescence in 7-10days. ${ }^{6-9}$ Rapid progression and continuous deterioration is suggestive of pancreatic necrosis. Continuous severe abdominal pain, continuous vomiting, paralytic ileus, positive symptoms of irritation of peritoneum and hemodynamic instability are the clinical signs suggestive of pancreatic necrosis. ${ }^{6-9}$ Pancreatic necrosis is divided in three periods on basis of progression. The first period is associated with hemodynamic instability and pancreatogenic shock. It lasts for initial 2-3days. There is violation of central hemodynamic, diminishment circulatory blood volume and microcirculation disorders. ${ }^{6}$ The second period (insufficiency of parenchymatous organs) lasts from $3^{\text {rd }}$ to the $7^{\text {th }}$ day of disease. There is involvement and dysfunction of multiple organ systems. The third period (post-necrosis complications) comes in 1-2weeks after the beginning of disease. Regenerative changes are para-pancreatic infiltrate, cysts and cystic fibrosis of pancreas. ${ }^{6}$

\section{Investigations}

Contrast-enhanced computed tomography (CECT) is the most reliable investigation to diagnose pancreatic necrosis. Necrosis appears as areas of low attenuation ( $<40$ to $50 \mathrm{HU})$ after injecting IV contrast (Figure 1). ${ }^{2}$ CECT allows accurate assessment of the extent of pancreatic necrosis and peri-pancreatic fluid collections. A CECT in the initial 3-4days of acute pancreatitis might underestimate or miss the necrosis. Usually, a CECT is advised if there is progressive clinical deterioration on medical management to find out any local complication. Serial CECT scans can also assess the progression of the disease and are an essential adjunct when surgical intervention is needed. ${ }^{2,10}$ Routine blood investigations reveal an increased hematocrit because of hypovolemia, a raised white cell count with leftward shift denoting the presence of inflammation, infection or both. ${ }^{2,3}$ Raised serum amylase levels ( $\geq 3$ times the upper limit of normal) are virtually diagnostic of acute pancreatitis. ${ }^{11}$ However the magnitude of this elevation does not correlate with the severity of disease. The levels rise within 2 to 12 hours of onset of symptoms and remain elevated up to 6days thereafter. Urinary amylase levels however, remain elevated for a relatively longer duration, being helpful in the diagnosis of patients who report a long time after the onset of symptoms. Serum lipase levels are also raised even after the serum amylase levels have returned to baseline. An ideal laboratory test for evaluation of acute pancreatitis (AP) should, in addition to accurately establishing the diagnosis, provide early assessment of its severity and identify the etiology. ${ }^{11,12}$ None of the available tests today meet all these criteria. In the diagnosis of AP, serum amylase and lipase remain important tests. Other enzymes for the diagnosis of AP-C-reactive protein, Phospholipase A2, Polymorph nuclear elastase, Immunoreactive trypsin, Interleukin-6, and Pancreatitisassociated protein are in experimental stage and have no clear role in the diagnosis and assessment of severity of AP..$^{11,12}$

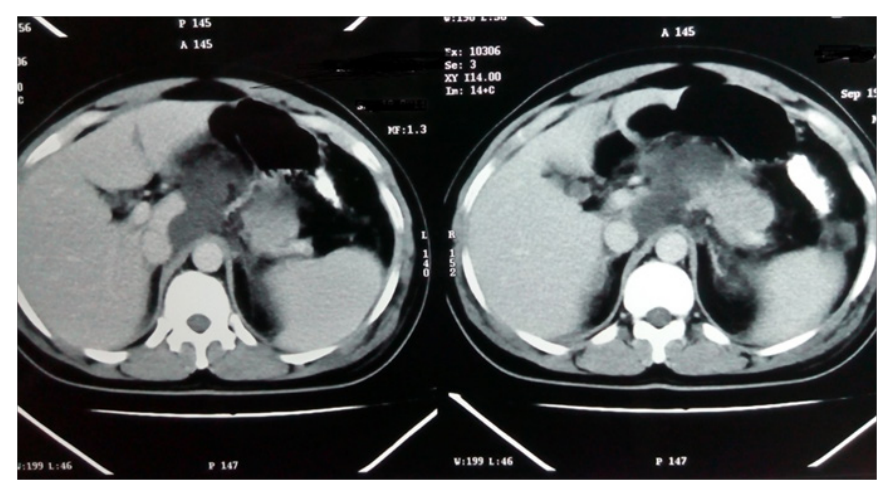

Figure I CT suggestive of pancreatic necrosis.

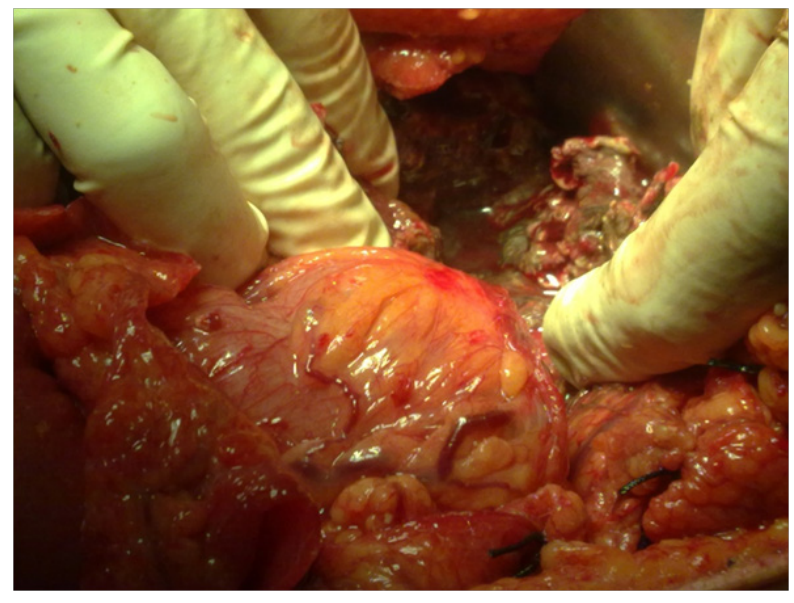

Figure 2 Open pancreatic necrosectomy showing necrosis.

\section{Factors predicting severity of pancreatitis}

Most of the patients suffering from mild attack of pancreatitis make an almost uneventful recovery. ${ }^{2,13}$ However, one third of the patients who have a severe affliction and who develop organ failure during the $1^{\text {st }}$ week, eventually develop necrosis involving more than $30 \%$ of the gland parenchyma. ${ }^{13}$ To decrease the mortality rate of the SAP, it is important to precisely evaluate the severity early in the disease and initiate appropriate treatment as early as possible., ${ }^{2,13}$ Several prognostic criteria have been designed to predict the severity and future course of an attack of acute pancreatitis. Out of these Imrie's scoring system ${ }^{14}$ and Ranson's criteria ${ }^{15}$ were widely used. Their use however, is limited by the fact that they can be accurately calculated only 48hours after hospitalization. ${ }^{16}$ The APACHE-II (Acute Physiology And Chronic Health Evaluation) score is preferable as it can be used at the time of admission and thereafter quantifies the disease severity continuously. An APACHE score of 8 or more indicates a severe disease with a relatively poorer outcome. But because of multiple factors being taken into account the calculation becomes cumbersome and time consuming. This fact downgrades the popularity of APACHE- II scoring system. ${ }^{16}$ Balthazar et al developed a radiological prognostic index, in which the severity of disease is 
estimated by number of pancreatic fluid collection and the extent of pancreatic non perfusion as visualized by contrast enhanced CT scan of the abdomen. ${ }^{17}$ It is a well established fact that a thorough clinical examination by an experienced clinician can actually surpass any of the above mentioned scoring systems. Also these scoring system have no diagnostic utility. Their use can be justified merely for triaging the patients and for the research purposes.

\section{Detection of infection}

Absence of infective organism in necrotic material is Sterile PN. ${ }^{18}$ Sterile necrosis has a better prognosis than infected necrosis. Mortality rate of sterile necrosis is near zero in the absence of systemic complications. ${ }^{18}$ However, mortality increased to $38 \%$ in patients with a single systemic complication. ${ }^{18,19}$ Patient without systemic complications and secondary infections can be managed with supportive care. A CT-guided FNA (Fine needle aspiration) is done in patient with systemic complications and/or suspected secondary infection to confirm or disprove infection, and patient without infection can be managed medically. Patient with severe systemic toxicity and high APACHE-II or Ranson's scores with progressive deterioration have a poor prognosis without aggressive surgical debridement. ${ }^{19}$ Current opinion is to avoid debridement in sterile necrosis unless progressive clinical deterioration on medical therapy or life-threatening systemic complications. ${ }^{18-19}$ The main complication associated with pancreatic necrosis is infection and risk of infection is directly related to the amount of necrosis. ${ }^{20}$ The incidence of infected necrosis in patients with necrotizing pancreatitis is around $30 \%$. The peak incidence of infected necrosis is between 2 and 4weeks after onset of disease ${ }^{19-20}$ Infection is usually due to translocation of enteric bacteria as a result of increased intestinal permeability following mucosal ischemia and reperfusion injury. Organisms implicated are usually gram-negative rods (e.g., Escherichia coli, Klebsiella and Pseudomonas spp.) and Enterococcus species.,21 Infection is suspected in patients with prolonged fever, elevated WBC count, or progressive clinical deterioration despite adequate medical therapy. CECT can confirm the diagnosis by showing intra-pancreatic or peri-

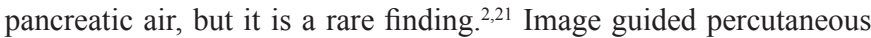
Fine-needle aspiration (FNA) should be done for Gram's stain and culture in suspected infective necrosis. False-negative results may occur with FNA. IV antibiotics should be started for infected pancreatic necrosis. Carbapenems are the choice and alternatives are quinolones, metronidazole, third-generation cephalosporins, and piperacillin. Antibiotics alone may not be effective in infected necrosis, which has a mortality of nearly $50 \%$ unless debrided surgically. ${ }^{2,19}$

\section{Prevention of infection of necrosis}

Bacterial translocation is believed to be the mechanism associated with infected pancreatic necrosis. ${ }^{2}$ Various strategies aimed at preventing bacterial translocation and subsequent infections are studied e.g.: antibiotics, probiotics, and enteral nutrition. Role of antibiotic prophylaxis to prevent infection in pancreatic necrosis is controversial. Most of the meta-analyses and current international guidelines did not support any significant beneficial effect of antibiotic prophylaxis on infection of pancreatic necrosis and mortality. ${ }^{22}$ Dellinger et al performed a prospective, double blinded randomized controlled study involving 100patients. Pancreatic or peripancreatic infections developed in 18\%(9 of 50) of patients in the meropenem group and $12 \%(6$ of 50$)$ in the placebo group $(\mathrm{P}=0.401)$. Overall mortality rate was $20 \%$ (10 of 50$)$ in the meropenem group compared with $18 \%(9$ of 50$)$ in the placebo group $(\mathrm{P}=0.799)$. Surgical intervention was required in $26 \%$ (13 of 50 ) and $20 \%(10$ of 50$)$ of the meropenem and placebo groups, respectively $(\mathrm{P}=0.476){ }^{23}$ This study suggested no statistically significant difference between the two groups and did not support role of prophylactic antibiotic use in severe acute pancreatitis. Isenmann demonstrated in a large randomized study that antibiotic prophylaxis has no beneficial effects with regard to the reduction of pancreatic infection and mortality. ${ }^{24}$

Probiotics are believed to prevent bacterial overgrowth, reinforce the mucosal barrier function and may reduce bacterial translocation. ${ }^{25}$ Early studies suggested role of probiotic in reduction of infected pancreatic necrosis. ${ }^{25} \mathrm{~A}$ large, recent study did not show any beneficial effect of an enterally administered multispecies probiotic mixture on the incidence of infections. However, there was increased mortality in probiotic group as compared with placebo group. Probiotics is currently not advised in severe acute pancreatitis. Enteral nutrition may have a beneficial role in preventing infections. ${ }^{26}$ Several studies supported that early starting of enteral feeding in compare to parenteral nutrition reduces mortality, multi-organ failure and systemic infections by reducing bacterial translocation. Studies reported that the simpler and cheaper nasogastric feeding appears to be well tolerated and is as safe as nasojejunal feeding in severe acute pancreatitis.

\section{Management}

\section{Conservative treatment}

Initial treatment is mainly supportive and focuses on adequate fluid resuscitation, pain management, regular monitoring of the clinical parameters and intensive monitoring for organ failure. ${ }^{2,13}$ Parenteral analgesics or patient-controlled analgesia are generally needed for severe pain. ${ }^{13}$ In condition of organ dysfunction or organ failure, intensive care units are preferred for supportive treatment. Warndorf MG reported that aggressive early fluid resuscitation to correct hypotension and hemo concentration is associated with decreased risk of systemic inflammatory response syndrome and organ failure. ${ }^{27}$ $\mathrm{Wu} \mathrm{BU}$ et al reported that fluid resuscitation with lactated Ringer's solution reduces the systemic inflammation compared with normal saline. ${ }^{28}$ About two-thirds of necrotizing pancreatitis remain sterile and can be successfully managed conservatively. An intervention is associated with risk of introducing infection to sterile necrosis (55\%$59 \%)^{29}$

\section{Invasive treatment}

Current standard indication for intervention is infected necrosis. Other indications include the drainage-debridement of persistent symptomatic fluid collections, and progressive deterioration with ongoing organ dysfunction in the absence of documented infection. ${ }^{29}$ Intervention should be delayed to approximately 3-4weeks after onset of disease. Broad-spectrum antibiotics and supportive therapy are used initially in infected necrosis to post-pone intervention. Delay lead to encapsulation and demarcation of peripancreatic collections, which decrease the complications such as bleeding and perforation. ${ }^{29,30}$ Decision for intervention is taken on basis of clinical condition and encapsulation of the infected collection rather than a positive FNA. ${ }^{31}$

\section{The step up approach}

Here, minimal invasive techniques are used initially with consideration of open surgery for non-responders. The first step is catheter drainage (i.e., image guided percutaneous, or endoscopic 
transluminal) of the infected collection to reduce sepsis and postpone or even avoid necrosectomy. If this fails, the next step is either surgical or endoscopic minimally invasive necrosectomy. The PANTER trial (Minimally Invasive Step up Approach versus Maximal Necrosectomy in Patients) randomized 88 patients to open surgical debridement versus the step-up approach consisting of percutaneous drainage followed by minimally invasive VARD if necessary. Mortality rates were almost identical (about 20\%) in both group but major complications were less in step up approach (70 vs. $40 \%){ }^{32}$ Various procedures included in Step-up approach are mentioned below.

\section{Minimally invasive approaches}

There is recent interest in minimal invasive approaches due to surgical trauma associated with open surgery. ${ }^{33}$ Two-hit response hypothesis states that many patients with a SAP will demonstrate an inappropriate and exaggerated inflammatory response to a second traumatic challenge. Thus a second hit, for example from an open necrosectomy, may lead to an overwhelming systemic inflammatory response and high mortality. Minimal invasive procedures produce less surgical stress in compare to open necrosectomy. Various approaches include percutaneous catheter drainage (PCD), endoscopic transluminal drainage (ETD), endoscopic transluminal necrosectomy $(\mathrm{ETN})$, and minimally invasive retroperitoneal surgical necrosectomy. ${ }^{33-36}$

\section{Image guided percutaneous catheter drainage}

It drains the infected fluid (i.e., pus) under pressure, without removal of necrotic material. It is feasible in $>95 \%$ of infected necrotizing pancreatitis, often via a left-sided retroperitoneal approach. ${ }^{34,35}$ In a recent systematic review, more than half of the cases of pancreatic necrosis were successfully treated with PCD alone and thus avoiding an additional necrosectomy. PCD may also used initially to improve clinical condition followed by surgery in later period when encapsulation of the necrotic collections has occurred.

\section{Minimally invasive retroperitoneal necrosectomy}

Most commonly used techniques are sinus tract endoscopy, laparoscopic transabdominal necrosectomy and video-assisted retroperitoneal debridement (VARD) ${ }^{36}$

\section{Endoscopic transluminal drainage and necrosectomy}

In ETD, endoscopic ultrasound is used to determine the extent of necrosis and the optimal site of drainage. After that, the collection is punctured endoscopically through the gastric or duodenal wall, followed by balloon dilatation of the tract. ${ }^{37}$ For continuous postoperative irrigation, double-pigtail stents and a nasocystic catheter are used. Several studies reported complication rates of $2 \%$ $21 \%$ and mortality rates of $0 \%-6 \%$ with these procedures. With no improvement or deterioration after ETD, ETN can be done to remove infected necrosis. ${ }^{37}$

\section{Primary open necrosectomy}

This is invasive surgical debridement of infected necrosis leading to future pancreatic insufficiency. ${ }^{31,38}$ Following laparotomy the lesser sac is entered, colon is mobilised downwards and the pancreas is identified. Debridement is done by blunt finger dissection and wide bore suction drainage. Large drains and irrigating catheters are placed within the pancreatic bed and irrigation is continued post surgery. It is associated with a high morbidity (34\%-95\%) and mortality (11\%$39 \%$ ). Outcomes are dependent on time, surgery in the first 14days having a mortality rate of $75 \%$; surgery between 15 and 29 days and after 30days having mortality rates of $45 \%$ and $8 \%$, respectively.

\section{Conclusion}

Pancreatic necrosis is a challenging complication of severe acute pancreatitis and is associated with significant morbidity and mortality. APACHE- II and Ranson's criteria are useful to predict development of SAP. CECT is most reliable non-invasive diagnostic method for pancreatic necrosis. Initial management of sterile pancreatic necrosis is conservative with adequate fluid resuscitation and pain management. Conservative management is successful in two-third of sterile necrosis. Currently, there is no proven role of antibiotics and probiotics in prevention of infection in necrosis. Early enteral feeding is beneficial in preventing infection in necrosis. Around 30\% of sterile necrosis spontaneously develop infection and need an invasive intervention. Ideal time for intervention in infected necrosis is usually $3-4$ weeks after onset of symptoms when adequate encapsulation and demarcation of infected necrosis have been occurred. A minimal invasive step-up approach is associated with less morbidity and identical mortality in compare to open necrosectomy.

\section{Acknowledgements}

None.

\section{Conflicts of interest}

The author declares on conflict of interest.

\section{References}

1. Brunschot SV, Bakker OJ, Besselink MG, et al. Treatment of necrotizing pancreatitis. Clinical Gastroenterology and Hepatology. 2012;10(11):1190-1201.

2. Eric HJ, Daniel BC, Waddah BA, et al. Exocrine Pancreas. In: Townsend CM, et al. editors. Philadelphia; 2012. p. 1515-1531.

3. Beger HG, Rau BM. Severe acute pancreatitis: Clinical course and management. World J Gastroenterol. 2007;13(38):5043-5051.

4. Saluja AK, Lerch MM, Phillips PA. Why does pancreatic overstimulation cause pancreatitis. Anпu Rev Physiol. 2007;69:249-269.

5. Whitcomb DC. Clinical practice, Acute pancreatitis. $N$ Engl $J$ Med. 2006;354(20):2142-2215.

6. Banks PA. Epidemiology, natural history, and predictors of disease outcome in acute and chronic pancreatitis. Gastrointest Endorse. 2002;56(6Suppl):S226-230.

7. Bollen TL, Besselink MG, Santvoort HC, et al. Toward an update of the Atlanta classification on acute pancreatitis: review of new and abandoned terms. Pancreas. 2007;35(2):107-113.

8. Vege SS, Chari ST. Organ failure as an indicator of severity of acute pancreatitis: time to revisit the Atlanta classification. Gastroenterology. 2005;128(4):1133-1135.

9. Whitcomb DC, Yadav D, Adam S. Multicenter approach to recurrent acute and chronic pancreatitis in the United States: the North American Pancreatitis Study 2 (NAPS2). Pancreatology. 2008;8(4-5):520-531.

10. Spanier BW, Nio Y, Van der Hulst RW. Practice and yield of early CT scan in acute pancreatitis: a Dutch observational multicenter study. Pancreatology. 2010;10(2-3):222-228. 
11. Winslet M, Hall C, London NJ, et al. Relation of diagnostic serum amylase levels to etiology and severity of acute pancreatitis. Gut. 1992;33(7):982 986.

12. Yadav D, Agarwal N, Pitchumoni CS. A critical evaluation of laboratory tests in acute pancreatitis. Am J Gastroenterol. 2002;97(6):1309-1318

13. Slavin J, Ghaneh P, Sutton R. Management of necrotizing pancreatitis. World J Gastroenterol. 2001;7(4):476-781.

14. Imrie CW, Benjamin IS, Ferguson JC. A single centre double blind trial of trasylol therapy in primary acute pancreatitis. Br J Surg. 1978;65:337-341.

15. Ranson JH, Rifkind KM, Roses DF. Prognostic signs and the role of operative management in acute pancreatitis. Surg Gynecol Obstet. 1974;139:69-81.

16. Larvin M, McMahon M. APACHE-II score for assessment and monitoring of acute pancreatitis. Lancet. 1989;2(8665):201-205.

17. Balthazar EJ. Acute pancreatitis: Assessment of severity with clinical and CT evaluation. Radiology. 2002;223(3):603-613.

18. Perez A, Whang EE, Brooks DC. Is severity of necrotizing pancreatitis increased in extended necrosis and infected necrosis. Pancreas. 2002;25(3):229-233.

19. Boland B, Colquhooun S, Menon V. Current surgical management of infected pancreatic necrosis. Amer Surg. 2010;76:1096-1099.

20. Schneider L, Buchler MW, Werner J. Acute pancreatitis with an emphasis on infection. Infect Dis Clin North Am. 2010;24(4):921-941, viii

21. Van Santvoort HC, Bakker OJ, Bollen TL. A conservative and minimally invasive approach to necrotizing pancreatitis improves outcome. Gastroenterology. 2011;141:1254-1263.

22. Wittau M, Mayer B, Scheele J. Systematic review and meta-analysis of antibiotic prophylaxis in severe acute pancreatitis. Scand J Gastroenterol. 2011;46:261-270.

23. Dellinger EP, Tellado JM, Soto NE, et al. Early antibiotic treatment for severe acute necrotizing pancreatitis: a randomized, double-blind, placebo-controlled study. Ann Surg. 2007;245(5):674-683.

24. Isenmann R, Rünzi M, Kron M. Prophylactic antibiotic treatment in patients with predicted severe acute pancreatitis: a placebo-controlled, double-blind trial. Gastroenterology. 2004;126(4):997-1004.

25. Van Santvoort HC, Besselink MG, Timmerman HM. Probiotics in surgery. Surgery. 2008;143:1-7.
26. Al Omran M, Albalawi ZH, Tashkandi MF. Enteral versus parenteral nutrition for acute pancreatitis. Cochrane Database Syst Rev. 2010;20(1):CD002837.

27. Warndorf MG, Kurtzman JT, Bartel MJ. Early fluid resuscitation reduces morbidity among patients with acute pancreatitis. Clin Gastroenterol Hepatol. 2011;9(8):705-709.

28. Wu BU, Hwang JQ, Gardner TH. Lactated Ringer's solution reduces systemic inflammation compared with saline in patients with acute pancreatitis. Clin Gastroenterol Hepatol. 2011;9(8):710-717.

29. Büchler MW, Gloor B, Müller CA, et al. Acute necrotizing pancreatitis: treatment strategy according to the status of infection. Ann Surg. 2000;232(5):619-626.

30. Mier J, León EL, Castillo A, et al. Early versus late necrosectomy in severe necrotizing pancreatitis. Am J Surg. 1997;173(2):71-75.

31. Rodriguez JR, Razo AO, Targarona J. Debridement and closed packing for sterile or infected necrotizing pancreatitis: insights into indications and outcomes in 167 patients. Ann Surg. 2008;247(2):294-299.

32. Van Santvoort HC, Besselink MG, Bakker OJ. A step-up approach or open necrosectomy for necrotizing pancreatitis. N Engl J Med. 2010;362:14911502 .

33. Windsor JA. Minimally invasive pancreatic necrosectomy. Br J Surg. 2007;94:132-133.

34. Shankar S, Sonnenberg E, Silverman SG, et al. Imaging and percutaneous management of acute complicated pancreatitis. Cardiovasc Intervent Radiol. 2004;27(6):567-580.

35. Van Baal MC, Santvoort HC, Bollen TL. Systematic review of percutaneous catheter drainage as primary treatment for necrotizing pancreatitis. Br J Surg. 2011;98(1):18-27.

36. Raraty MG, Halloran CM, Dodd S. Minimal access retroperitoneal pancreatic necrosectomy: improvement in morbidity and mortality with a less invesive approach. Ann Surg. 2010;251(5):787-793.

37. Bakker OJ, Santvoort HC, Brunschot S. Endoscopic transgastric vs. surgical necrosectomy for infected necrotizing pancreatitis: a randomized trial. JAMA. 2012;307:1053-1061.

38. Traverso LW, Kozarek RA. Pancreatic necrosectomy: definitions and technique. J Gastrointest Surg. 2005;9(3):436-439. 